University of Nebraska - Lincoln

DigitalCommons@University of Nebraska - Lincoln

8-1992

\title{
Solutions of the heat conduction equation in multilayers for photothermal deflection experiments
}

\author{
William A. McGahan \\ University of Nebraska-Lincoln \\ Kevin D. Cole \\ University of Nebraska-Lincoln, kcole1@unl.edu
}

Follow this and additional works at: https://digitalcommons.unl.edu/mechengfacpub

Part of the Mechanical Engineering Commons

McGahan, William A. and Cole, Kevin D., "Solutions of the heat conduction equation in multilayers for photothermal deflection experiments" (1992). Mechanical \& Materials Engineering Faculty Publications. 36.

https://digitalcommons.unl.edu/mechengfacpub/36

This Article is brought to you for free and open access by the Mechanical \& Materials Engineering, Department of at DigitalCommons@University of Nebraska - Lincoln. It has been accepted for inclusion in Mechanical \& Materials Engineering Faculty Publications by an authorized administrator of DigitalCommons@University of Nebraska Lincoln. 


\title{
Solutions of the heat conduction equation in multilayers for photothermal deflection experiments
}

\author{
William A. McGahan \\ Center for Microclectronic and Optical Materials Research and Departments of Electrical Engineering \\ and Physics, University of Nebraska-Lincoln, Lincoln, Nebraska 68588-0511 \\ K. D. Cole \\ Department of Mechanical Engineering, University of Nebraska-Lincoln, Lincoln, Nebraska 68588-0511
}

(Received 7 October 1991; accepted for publication 4 May 1992)

\begin{abstract}
Exact expressions are presented for the deflection of a laser beam passing parallel to and above the surface of a sample heated by a periodically modulated axisymmetric laser beam. The sample may consist of any number of planar films on a thick substrate. These exact expressions are derived from a local Green's function treatment of the heat conduction equation, and contain an exact analytical treatment of the absorption of energy in the multilayered system from the heating laser. The method is based on calculation of the normal component of the heat fluxes across the layer boundaries, from which either the beam deflections or the temperature anywhere in space can be easily found. A central part of the calculation is a tridiagonal matrix equation for the $N+1$ normal boundary fluxes, where $N$ equals the number of films in the sample, with the beam deflections given as simple functions of the normal heat flux through the top surface of the sample. Even though any layer or layers in the sample (including the substrate) can be optically absorbing, the final results are remarkably simple both in form and ease of calculation, even for large numbers of layers. In the case of an infinitesimal probe beam, the beam deflections are given by an expression involving a single numerical integration which can be eliminated for data analysis by Fourier transforming the experimental data. A general expression for the measured signals for the case of four-quadrant detection is also presented and compared to previous calculations of detector response for finite probe beams.
\end{abstract}

\section{INTRODUCTION}

The measurement of thermal properties of thin-film media is an important issue in magneto-optical recording, ion implantation, electron-beam lithography, and many other arcas of materials science and processing. The present paper is concerned with photothermal deflection methods for the measurement of thermal properties of thin films. Photothermal deflection (PTD) experiments are a powerful tool for the measurement of thermal properties (conductivity, diffusivity) of both bulk materials and thin films. ${ }^{1,2}$ In these experiments, a periodically modulated laser beam excites thermal waves in the sample which in turn heat the ambient region above the sample. The resulting gradient of the temperature (and hence the index of refraction) in the ambient above the sample can be measured by detecting the deflection of a second laser beam passing above the sample and parallel to the sample surface. The probe beam is initially centered on a four-quadrant silicon photodetector, such that deflection signals can be measured as differences in voltage between the upper and lower or left- and righth-land-side halves of the detector. The ambient medium through which the probe beam passes is usually air, but other gases or fluids can be used to increase the deflection signal. The deflection of the probe beam is measured synchronously with respect to the chopping of the heating laser beam, and has components parallel and normal to the surface of the sample, each of which has components both in and out of phase with the modulation of the heating laser beam. Nonlinear regression is used to find values of the sample thermal properties and experi- mental parameters such that calculated probe beam deflections match the experimental deflections as closely as possible. The calculation of probe beam deflections, then, is an important part of the PTD measurement technique.

For small temperature changes, the angular deflection of an infinitesimally thin probe beam passing through a region of nonuniform temperature is given by $^{3}$

$$
\mathbf{M}=-\int \frac{1}{n} \frac{d n}{d T} \nabla T(\mathbf{r}) \times d \mathbf{r}
$$

where $n$ is the index of refraction of the ambient medium, and the integral is evaluated along the path of the probe beam. This expression can be readily evaluated once the temperature distribution in the ambient (at least along the beam path) is known.

Our research in PTD theory is motivated by our work with PTD measurements on multilayers. We are particularly interested in measuring the thermal conductivity of thin films used for magneto-optical recording media. Currently, the most widely used magneto-optical media are rare-earth/transilion-Inetal-based alloys, which are highly reactive in atmosphere and require protective coatings to preserve film quality. Thin-film samples of these materials would necessarily consist of at least two or three films on a thick substrate, hence a multilayer formalism is required for analysis of the experimental data. Also, we are interested in dielectric films with low optical absorption. For this reason we also require an exact optical model to treat the distribution of heat absorbed within the dielectric, as well as within an additional absorbing layer or substrate. 
To our knowledge, there is no PTD theory in the literature that combines an exact treatment of optical absorption, axisymmetric laser heating, multiple layers, and ease of calculation, which is important if the theory is to be used for regression analysis of data. Individual parts of a complete theory for multilayers are available in the literature of PTD theory, and we discuss pertinent literature below.

There is an extensive literature concerning theoretical temperature prediction in multilayer structures heated by laser beams. Since the initial calculations by $\operatorname{Lax}^{4}$ for a semi-infinite body with steady heating, there have been several predictive multilayer temperature calculations, subject to varying degrees of approximation. Burgener and Reedy ${ }^{5}$. studied a two-layer structure with a continuous-wave scanned laser beam, and they indicated how to apply their method to $N$ layers; however, laser heating was limited to complete absorption at the surface of a single film. Ander$\operatorname{son}^{6}$ included linearly varying absorption inside the material. Actual exponential absorption could be approximated by several piecewise-linear absorbing layers. Vaez Iravani and Wickramasinghe ${ }^{7}$ used the temporal Fourier transform to find the temperature in such a multilayer structure. The temperature expressionis within each layer were linked to the adjacent layers by discontinuity conditions on temperature and heat flux at the layer interfaces. Kant ${ }^{8}$ studied the same absorption geometry but used the Laplace transform appropriate for the temperature rise caused by a single laser pulse.

An important application for temperature calculations in multilayers is modeling the thermomagnetic write process in magneto-optical recording media. Typically the geometry of interest is one or more transparent layers on top of one opaque (high absorption) layer, so the laser heating is limited to complete absorption in a region of infinitesimal thickness. Madison and McDaniel, ${ }^{9}$ in the most ambitious temperature theory to date, analyzed a scanned laser beam with arbitrary absorption across one layer, for a single transient pulse of the laser beam. They use globally defined Green's functions combined with Laplace transform methods to obtain their solution, and they indicate how to extend the method to $N$ layers. As $N$ increases the Green's function for the system becomes increasingly complex.

There is also extensive existing literature concerning PTD experiments and related theory: Mandelis and Royce $^{10}$ employed a one-dimensional model to calculate photoacoustic effects for a thin sample on a thick backing layer, with exponential absorption of heat in the thin sample layer only. Jackson ${ }^{-}$et al. ${ }^{11}$ calculated deflections for one-layer systems, in which only the film was optically absorbing; and extended their calculation to include finite probe beam corrections for four-quadrant detection. Aamodt and Murphy ${ }^{12}$ considered both bulk and singlelayered structures and found expressions for the beam deflections for several limiting cases, as well as a finite probe beam correction for the case of position sensitive detectors. The calculation by Grosse and Wynands ${ }^{13}$ of photoacoustic IR spectra for multilayers is of particular interest, as they calculate the energy absorbed by the multilayer from the heating laser beam exactly. Their calculation is one dimensional, however, and requires numerical convolution over the heating distribution in the sample. Finally, Reichling, Grönbeck, and Schneider ${ }^{14}$ have recently published calculations for systems consisting of two layers on a thick substrate with exponential absorption in both layers or in one layer and the substrate. Their solution is based on the Hankel transform technique of Jackson et al., ${ }_{-}$- and allows for the inclusion of a thermal resistance between the two layers. They present plots of calculations performed with this formalism, but do not present the actual theory used for the calculations.

The remainder of the paper is structured as follows: In Sec. II we show the necessary Green's functions for both layers and semi-infinite regions, and apply Fourier and Hankel transformations to these functions. In Sec. III we treat the absorption of energy from the heating laser beam in multilayer systems analytically, and derive simple expressions for the volume integrals in the Green's function temperature expressions. In Sec. IV we derive temperature solutions for the two-body case in which the sample is assumed to be infinitely thick. In Sec. V we extend the two-body solution to systems with any number of layers. Section VI contains the derivation of simple expressions for probe beam deflections in PTD experiments based on the temperature solutions presented previously. Section VII presents a derivation of the expressions for the signals measured in PTD experiments using four-quadrant detectors. Section VIII contains sample calculations demonstrating the usefulness of the theory, and Sec. IX summarizes this work and presents some conclusions.

\section{CALCULATION OF GREEN'S FUNCTIONS}

We will consider the case of an arbitrary number of films on a thermally and optically thick substrate. The geometry of the problem is shown in Fig. 1. Note that each

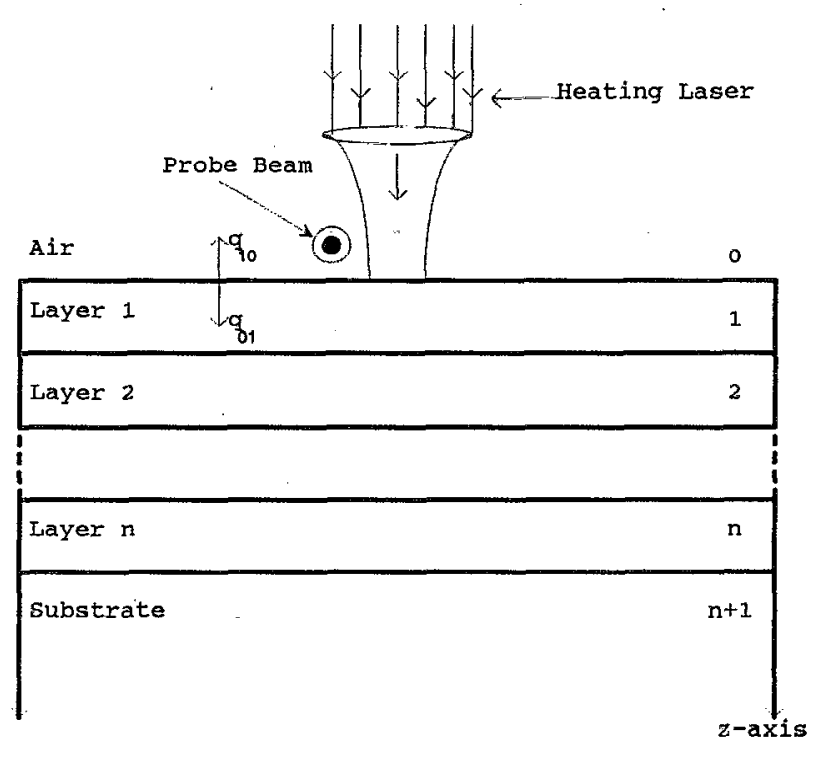

FIG. 1. Geometry for the photothermal deflection experiment. 
region is given a numeric index ( 0 for the ambient, 1 for the first layer, etc.), and all quantities that refer to a specific region will be subscripted with the number that indexes that region in Fig. 1. Also, we will use a local $z$ coordinate in each region. For example, in any layer and in the substrate we fix $z=0$ to be at the top of the layer, with the positive $z$ direction being downward into the sample. In the ambient region above the sample we fix $z=0$ at the ambient/sample interface with the positive $z$ direction being upward, away from the sample. This choice of local coordinates simplifies the task of matching solutions in each region to those of neighboring regions at the mutual boundaries.

We seek solutions to the following axisymmetric heat conduction equation:

$\frac{1}{r} \frac{\partial}{\partial r}\left(r \frac{\partial T(\bar{r}, z, t)}{\partial r}\right)+\frac{\partial^{2} T(r, z, t)}{\partial z^{2}}-\frac{1}{\alpha} \frac{\partial T(r, z, t)}{\partial t}=-\frac{g(r, z, t)}{\kappa}$,

where $\alpha$ is the thermal diffusivity, $\kappa$ is the thermal conductivity, and $g(r, z, t)$ is the power per unit volume transferred to the medium at $(r, z, t)$ by the modulated laser beam. The thermal conductivity and diffusivity are assumed to be independent of both radius and temperature. This is equivalent to assuming the sample is laterally homogeneous and that the rise of temperature induced by the laser heating is small enough that the sample thermal properties are constant over the resultant range of temperatures. We will find separate solutions in each homogeneous region (substrate, film, air), and enforce continuity of both temperature and normal components of the heat flux at the boundaries. We will also require that the temperature elevation go to zero at points far removed from the region of heating.

The Green's function $G\left(r, z, t \mid r^{\prime}, z^{\prime}, \tau\right)$ for this problem is defined as the solution of Eq. (2) with the source term replaced by a $\delta$-function source located at $\left(r^{\prime}, z^{\prime}, \tau\right)$ :

$\frac{1}{r} \frac{\partial}{\partial r}\left(r \frac{\partial G}{\partial r}\right)+\frac{\partial^{2} G}{\partial z^{2}}-\frac{1}{\alpha} \frac{\partial G}{\partial t}=-\frac{\delta\left(r-r^{\prime}\right) \delta\left(z-z^{\prime}\right) \delta(t-\tau)}{2 \pi \alpha r^{\prime}}$,

subject to the requirement that the normal derivative of $G$ vanishes on the boundaries of the region of interest. Separate Green's functions are required for semi-infinite regions and for layers. Beck et al. have found solutions of Eq. (3) of the form, ${ }^{15}$ for semi-infinite, $\partial G / \partial z=0$ at $z=0$,

$$
\begin{aligned}
G\left(r, z, t \mid r^{\prime}, z^{\prime}, \tau\right)= & \frac{1}{4 \pi^{3 / 2}} \frac{1}{\sqrt{\alpha(t-\tau)}}\left[\exp \left(-\frac{\left(z-z^{\prime}\right)^{2}}{4 \alpha(t-\tau)}\right)\right. \\
& \left.+\exp \left(-\frac{\left(z+z^{\prime}\right)^{2}}{4 \alpha(t-\tau)}\right)\right] \\
& \times \int_{0}^{\infty} \exp \left[-\beta^{2} \alpha(t-\tau)\right] \beta J_{0}(\beta r) \\
& \times J_{0}\left(\beta r^{\prime}\right) d \beta,
\end{aligned}
$$

for $t>\tau$, zero otherwise; and for layer, $\partial G / \partial z=0$ at both $z=0$ and at $z=d$,

$$
\begin{aligned}
G\left(r, z, t \mid r^{\prime}, z^{\prime}, \tau\right)= & \frac{1}{2 \pi d}\left[1+2 \sum_{m=1}^{\infty} \cos \left(\frac{m \pi z}{d}\right)\right. \\
& \left.\times \cos \left(\frac{m \pi z^{\prime}}{d}\right) \exp \left(-\frac{m^{2} \pi^{2} \alpha(t-\tau)}{d^{2}}\right)\right] \\
& \times \int_{0}^{\infty} \exp \left[-\beta^{2} \alpha(t-\tau)\right] \beta J_{0}(\beta r) \\
& \times J_{0}\left(\beta r^{\prime}\right) d \beta
\end{aligned}
$$

for $t>\tau$, zero otherwise, where $J_{0}(x)$ is the zeroth-order Bessel function, and $d$ denotes the thickness of the layer.

The temperature at a point $(r, z, t)$ in space time is then given by the sum of two integrals,

$$
\begin{aligned}
T(r, z, t)= & \frac{\alpha}{\kappa} \int_{S} \int_{\tau=-\infty}^{t} q\left(r^{\prime}, \tau\right) G\left(r, z, t \mid r^{\prime}, z^{\prime}=z_{s}, \tau\right) \\
& \times 2 \pi r^{\prime} d r^{\prime} d \tau+\frac{\alpha}{\kappa} \int_{V} \int_{\tau=-\infty}^{t .} g\left(r^{\prime}, z^{\prime}, \tau\right) \\
& \times G\left(r, z, t \mid r^{\prime}, z^{\prime}, \tau\right) 2 \pi r^{\prime} d r^{\prime} d z^{\prime} d \tau
\end{aligned}
$$

where $S$ is the surface of the region within which $(r, z)$ is located, with differential area $2 \pi r^{\prime} d r^{\prime}$ and $V$ is the volume of the region with differential volume $2 \pi r^{\prime} d r^{\prime} d z^{\prime}$. We assume that all regions of interest are bounded by planes of constant $z^{\prime}$, and we denote the locations of the boundaries then by $z^{\prime}=z_{s}$. The quantity $q\left(r^{\prime}, \tau\right)$ is the normal component of the heat flux through the boundary of the region at $\left(r^{\prime}, z_{s}, \tau\right)$. The first integral in Eq. (5) is a surface integral, to be evaluated over the boundary surface of the region of interest. The second integral is a volume integral which is taken over the entire volume of the region. Physically, the second integral represents changes in temperature caused by heat generated within the region of interest, whereas the first integral represents changes in temperature due to heat generated outside the region which diffuses into the region of interest across the boundaries. It is the unknown heat fluxes $q\left(r^{\prime}, \tau\right)$ that we will determine by enforcing continuity of temperature and normal components of the heat fluxes at the boundaries. Note that since the Green's functions in Eq. (5) are nonzero only for $t>\tau$, the upper limit of the time integrations can be taken as $t$.

As we are interested only in situations where the volume heating source $g\left(r^{\prime}, z^{\prime}, t\right)$ is periodic in time (i.e., modulated laser beam) we will Fourier transform Eq. (5) to select the temperature response at the fundamental frequency of modulation. The use of lock-in detection in the actual PTD experiment allows us to effectively measure only the component of the beam deflections at the fundamental frequency of modulation. In addition, we apply Hankel transformations to Eqs. (4) and (5) to eliminate difficulties associated with the Bessel functions in Eq. (4). The Fourier- and Hankel-transformed temperature is then 


$$
\begin{aligned}
T(\beta, z, f)= & \frac{\alpha}{\kappa} \int_{S} q\left(r^{\prime}, f\right) G\left(\beta, z, f \mid r^{\prime}, z^{\prime}=z_{s}\right) 2 \pi r^{\prime} d r^{\prime} \\
& +\frac{\alpha}{\kappa} \int_{V} g\left(r^{\prime}, z^{\prime}, f\right) G\left(\beta, z, f \mid r^{\prime}, z^{\prime}\right) 2 \pi r^{\prime} d r^{\prime} d z^{\prime}
\end{aligned}
$$

where $G\left(\beta, z, f \mid r^{\prime}, z^{\prime}\right)$ is the Hankel transform of the Fourier transform of the Green's functions as given in Eq. (4). Note that the temperature in Eq. (6) now has units of (K) (length) ${ }^{2}$ (time) due to the integral transformations. The transformed Green's functions in Eq. (6) are given by, for semi-infinite $(0<z<\infty)$,

$$
\begin{aligned}
G\left(\beta, z, f \mid r^{\prime}, z^{\prime}\right)= & \frac{1}{4 \pi \alpha \eta}\left[\exp \left(-\eta\left|z-z^{\prime}\right|\right)\right. \\
& \left.+\exp \left(-\eta\left|z+z^{\prime}\right|\right)\right] J_{0}\left(\beta r^{\prime}\right),
\end{aligned}
$$

and for layer $(0 \leqslant z \leqslant d)$,

$$
\begin{aligned}
G\left(\beta, z, f \mid r^{\prime}, z^{\prime}\right) \\
=\frac{1}{2 \pi d \alpha}\left(\frac{1}{\eta^{2}}+2 \sum_{m=1}^{\infty} \frac{\cos \left(m \pi z^{\prime} / d\right) \cos (m \pi z / d)}{\eta^{2}+m^{2} \pi^{2} / d^{2}}\right) \\
\quad \times J_{0}\left(\beta r^{\prime}\right)
\end{aligned}
$$

where

$$
\eta \equiv \sqrt{\beta^{2}+2 \pi j f / \alpha}
$$

Other series expansions for Eq. (7b) exist, ${ }^{15}$ but we have chosen the particular form shown because all resulting integrations can be performed analytically. Also, unless the layer thickness $d$ becomes extremely large, the series in Eq. (7b) converges very rapidly. The expressions (6) and (7) yield the one-dimensional case when taken in the limit $\beta \rightarrow 0$. With the Green's functions given in Eq. (7), we now have the basic tools required to calculate the temperature and the photothermal beam deflections in arbitrary multilayered samples.

\section{CALCULATION OF VOLUME HEATING TERM}

In this section we calculate the contribution to the temperature caused by volume heating in each layer and the substrate for the case of an axisymmetric periodic laser heating beam. We first evaluate the energy per unit volume absorbed by the sample as a function of position in the sample using a procedure similar to that used in the onedimensional photoacoustic calculations of Grosse and Wynands. ${ }^{13}$ In a material with nonzero absorption in which a time-harmonic electromagnetic disturbance exists, the time-averaged energy absorbed per unit volume at a point $(r, z)$ is given by ${ }^{16}$

$$
\begin{aligned}
g(r, z, f)=\frac{1}{2} \operatorname{Re}\left(\mathbf{J}^{*} \cdot \mathbf{E}\right) & =\frac{\epsilon_{2} c}{2 \pi \lambda}|E(r, z)|^{2} \delta\left(f-f_{c}\right) \\
& =\frac{n k c}{\pi \lambda}|E(r, z)|^{2} \delta\left(f-f_{c}\right),
\end{aligned}
$$

where $\mathbf{J}$ is the electromagnetic current density at $(r, z), \mathbf{E}$ is the electric field at $(r, z), \epsilon_{2}$ is the imaginary part of the dielectric constant at $(r, z), n$ is the index of refraction, $k$ is the extinction coefficient, $c$ is the speed of light, $\lambda$ is the wavelength of the heating laser, and $f_{c}$ is the chopping frequency. Assuming a Gaussian intensity distribution for the radial dependence of $g(r, z, f)$ and normalizing appropriately yields

$g(r, z, f)=\frac{n k P_{0}}{\pi \lambda r_{h}^{2}} \exp \left(-\frac{r^{2}}{r_{h}^{2}}\right)\left|E_{\text {norm }}(z)\right|^{2} \delta\left(f-f_{c}\right)$,

where $P_{0}$ is the total power of the heating laser and $r_{h}$ is the Gaussian radius of the heating beam. We will henceforth suppress the $\delta$ function $\delta\left(f-f_{c}\right)$ in Eq. (9), and simply carry along the appropriate units (seconds). In Eq. (9), $E_{\text {norm }}(z)$ is the normalized and dimensionless electric field in the sample at $z$ due to an incident plane wave of unit amplitude. For a given multilayer structure, we use the method of Crook ${ }^{17}$ to calculate $E_{\text {norm }}(z)$. This method provides exact solutions for the electric field anywhere in the multilayer by requiring the total electric fields at each interface to satisfy the Fresnel reflection and transmission equations. In general, if we assume an incident beam of unit amplitude, consistent with Eq. (9), the dimensionless, normalized electric-field strength in any layer or the substrate is given by

$$
\begin{aligned}
E_{\text {norm }}(z)= & E_{+}(z=0) \exp \left(\frac{j 2 \pi n z}{\lambda}\right) \exp \left(-\frac{2 \pi k z}{\lambda}\right) \\
& +E_{-}(z=d) \exp \left(\frac{j 2 \pi n(d-z)}{\lambda}\right) \\
& \times \exp \left(-\frac{2 \pi k(d-z)}{\lambda}\right),
\end{aligned}
$$

where $\lambda$ is the wavelength of the heating laser beam. The quantities $E_{+}(z=0)$ and $E_{-}(z=d)$ refer to the (normalized) electric-field amplitudes of the downward propagating wave just below the top of the region $(z=0)$ and the upward propagating wave just above the bottom of the region $(z=d)$. This expression is exactly valid even when multiple reflections and interference effects occur, as the field quantities therein represent the sum of all upward and downward propagating waves in a layer, and are the natural result of calculations using Crook's method. In the substrate, where the region is only bounded at $z=0$, the second term in Eq. (10) does not exist. The energy transferred to the medium per unit volume [Eq. (8)] can then be evaluated from [Eqs. (8)-(10)] 


$$
\begin{aligned}
g\left(r^{\prime}, z^{\prime}, f\right)= & \frac{n k P_{0}}{\pi \lambda r_{h}^{2}} \exp \left(-\frac{r^{\prime 2}}{r_{h}^{2}}\right)\left[C_{1} \exp \left(-2 \gamma z^{\prime}\right)\right. \\
& \left.+C_{2} \exp \left(2 \gamma z^{\prime}\right)+C_{3} \cos \left(\delta d-2 \delta z^{\prime}+\phi\right)\right],
\end{aligned}
$$

where $C_{1}, C_{2}$, and $C_{3}$ are dimensionless constants,

$C_{1} \equiv\left|E_{+}\left(z^{\prime}=0\right)\right|^{2} \quad$ (semi-infinite or layer),

$C_{2} \equiv\left|E_{-}\left(z^{\prime}=d\right)\right|^{2} \exp (-2 \gamma d) \quad$ (layer only),

$C_{3} \equiv 2\left|E_{+}\left(z^{\prime}=0\right) E_{-}^{*}\left(z^{\prime}=d\right)\right| \exp (-\gamma d) \quad$ (layer only),

and

$\gamma \equiv 2 \pi k / \lambda, \quad \delta \equiv 2 \pi n / \lambda, \quad \phi \equiv \arg \left[E\left(z^{\prime}=0\right) E^{*}\left(z^{\prime}=d\right)\right]$.

In Eq. (11) there are three terms: The first term represents energy absorbed from the component of the beam propagating downward in the region, the second term represents energy absorbed from the upward propagating component of the beam, and the third term represents the effects of interference between the two components. Note that only the first term is nonzero for the semi-infinite substrate. Figure 2 shows the energy per unit volume absorbed in a single-layer system as a function of depth into the sample for a $1000-\AA$-thick layer with several different values of absorption in the layer. Equation (12) also accurately reproduces the results of calculations by Evans, Burgess, and McLean, ${ }^{18}$ which are based on evaluating the $z$ derivative of the power density in the medium.

We will now evaluate the volume integral in Eq. (6) for both the semi-infinite and layer cases. First, the volume heating integral in the substrate, which represents the change in temperature at $(\beta, z)$ due to all heat generated within the substrate, is given by

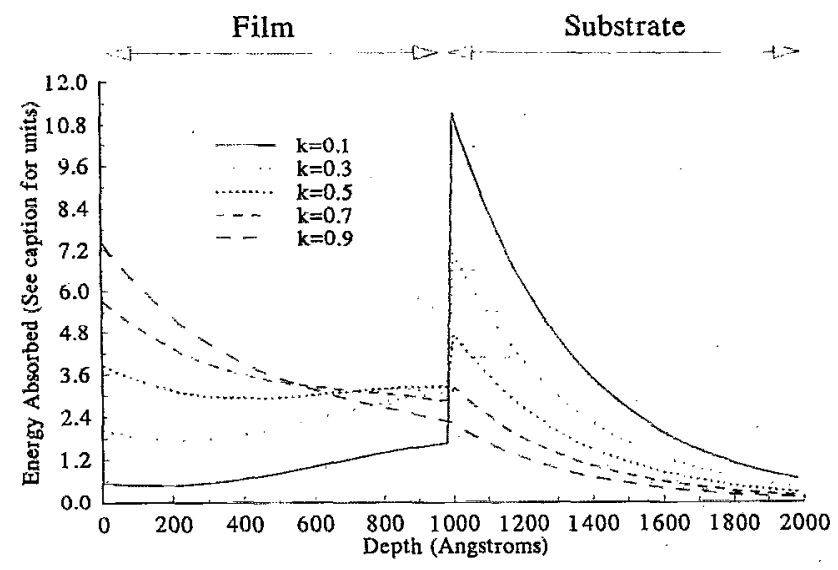

FIG. 2. Energy per unit volume $\left(\mathrm{J} / \mathrm{cm}^{3}\right) \times 10^{4}$ absorbed vs depth into the sample $(\AA)$ for a $1000 \AA$ layer on a thick substrate. For the substrate, $n=2.5, k=1.5$, and for the layer, $n=1.5, k=$ variable. Note the discontinuity at the film/substrate boundary $(z=1000 \AA)$. The incident laser power is $0.75 \mathrm{~mW}$, the laser wavelength is $6470 \AA$, and the profile is taken at the center of the heating beam.

$$
\begin{aligned}
B(\beta, z, f) \equiv & \frac{\alpha}{\kappa} \int_{0}^{\infty} 2 \pi r^{\prime} \int_{0}^{\infty} g\left(r^{\prime}, z^{\prime}, f\right) \\
& \times G\left(\beta, z, f \mid r^{\prime}, z^{\prime}\right) d z^{\prime} d r^{\prime} \\
= & \frac{n k P_{0} C_{1}}{2 \pi \lambda r_{h}^{2} \kappa \eta} \int_{0}^{\infty} r^{\prime} \exp \left(-\frac{r^{\prime 2}}{r_{h}^{2}}\right) J_{0}\left(\beta r^{\prime}\right) d r^{\prime} \\
& \times \int_{0}^{\infty}\left[\exp \left(-\eta\left|z-z^{\prime}\right|\right)\right. \\
& \left.+\exp \left(-\eta\left|z+z^{\prime}\right|\right)\right] \exp \left(-2 \gamma z^{\prime}\right) d z^{\prime} .
\end{aligned}
$$

Equation (13) can be readily evaluated ${ }^{19}$ to yield

$$
\begin{aligned}
B(\beta, z, f)= & \frac{n k P_{0} C_{1}}{4 \pi \lambda \eta \kappa} \exp \left(-\frac{\beta^{2} r_{h}^{2}}{4}\right) \\
& \times\left(\frac{\exp (-2 \gamma z)+\exp (-\eta z)}{\eta+2 \gamma}\right. \\
& \left.+\frac{\exp (-2 \gamma z)-\exp (-\eta z)}{\eta-2 \gamma}\right) .
\end{aligned}
$$

Similarly, the volume heating integral for a layer, with $z=0$ at the top of the layer and $z=d$ at the bottom of the layer, can be found:

$$
\begin{aligned}
B(\beta, z, f)= & \frac{n k P_{0}}{2 \pi \lambda d \kappa} \exp \left(-\frac{\beta^{2} r_{h}^{2}}{4}\right) \\
& \times\left[\frac{\mathbb{C}_{1}}{\eta^{2}}+2 \sum_{m=1}^{\infty}\left(\frac{\mathbb{C}_{m} \cos (m \pi z / d)}{\eta^{2}+m^{2} \pi^{2} / d^{2}}\right)\right],
\end{aligned}
$$

where

$$
\begin{aligned}
\mathbb{G}_{1} \equiv & \frac{C_{1}}{2 \gamma}[1-\exp (-2 \gamma d)]+\frac{C_{2}}{2 \gamma^{\prime}}[\exp (2 \gamma d)-1] \\
& +\frac{C_{3}}{\delta} \sin (\delta d) \cos (\phi),
\end{aligned}
$$

$$
\begin{aligned}
\mathbb{C}_{m} \equiv & \frac{2 \gamma}{4 \gamma^{2}+m^{2} \pi^{2} / d^{2}}\left\{C_{1}\left[1-(-1)^{m} \exp (-2 \gamma d)\right]\right. \\
& \left.+C_{2}\left[1+(-1)^{m} \exp (2 \gamma d)\right]\right\} \\
& +C_{3}\left(\frac{1}{2 \delta+m \pi / d}+\frac{1}{2 \delta-m \pi / d}\right) \\
& \times\left(\begin{array}{ll}
\sin (\delta d) \cos (\phi), & m=\text { even, } \\
\cos (\delta d) \sin (\phi), & m=\text { odd. }
\end{array}\right)
\end{aligned}
$$

Equations (14) and (15) represent the temperature change due to heat generated within a semi-infinite region and a layer, respectively. These expressions are given in the $\beta$ space of the Hankel transformation, as we will solve for temperature and beam deflections in this space. Given the volume heating [Eqs. (14)-(16)] and the Green's functions of the previous section [Eq. (7)], we may now solve for the temperature everywhere in and above the sample, and subsequently the photothermal beam deflections. 


\section{THE TWO-BODY SOLUTION}

We first turn to the solution of the two-body problem, corresponding to a thermally and optically thick sample with no films. The solutions for the temperature distribution in the ambient and the substrate are

$$
\begin{aligned}
T_{0}(\beta, z, f)= & \frac{\alpha_{0}}{\kappa_{0}} \int_{r^{\prime}=0}^{\infty} 2 \pi r^{\prime} q_{10}\left(r^{\prime}, f\right) \\
& \times G_{0}\left(\beta, z, f \mid r^{\prime}, z^{\prime}=0\right) d r^{\prime},
\end{aligned}
$$

for ambient, region 0 , and

$$
\begin{aligned}
T_{1}(\beta, z, f)= & \frac{\alpha_{1}}{\kappa_{1}} \int_{0}^{\infty} 2 \pi r^{\prime} q_{01}\left(r^{\prime}, f\right) G_{1}\left(\beta, z, f \mid r^{\prime}, z^{\prime}=0\right) \\
& \times d r^{\prime}+B_{1}(\beta, z, f),
\end{aligned}
$$

for subtrate, region 1 .

Two matching conditions are required to find the unknown heat fluxes $q_{10}$ and $q_{01}$. The first condition is that the normal component of the heat flux is continuous at the boundary $z=0$, which yields $q_{10}\left(r^{\prime}, f\right)=-q_{01}\left(r^{\prime}, f\right)$. The subscript $i j$ in $q_{i j}$ refers to heat passing from region $i$ into region $j$ (see Fig. 1). The second condition is that the temperatures $T_{0}$ and $T_{1}$ in Eq. (17) must be equal at the boundary $(z=0)$. After insertion of the Green's functions, the temperature matching condition yields

$$
\begin{aligned}
2 \pi \frac{\alpha_{0}}{\kappa_{0}} & \int_{0}^{\infty} r^{\prime} q_{10}\left(r^{\prime}, f\right)\left(\frac{1}{2 \pi \alpha_{0} \eta_{0}}\right) J_{0}\left(\beta r^{\prime}\right) d r^{\prime} \\
= & -\frac{2 \pi \alpha_{1}}{\kappa_{1}} \int_{0}^{\infty} r^{\prime} q_{10}\left(r^{\prime}, f\right)\left(\frac{1}{2 \pi \alpha_{1} \eta_{1}}\right) \\
& \times J_{0}\left(\beta r^{\prime}\right) d r^{\prime}+B_{1}(\beta, z=0, f),
\end{aligned}
$$

or

$$
\begin{aligned}
& \left(\frac{1}{\kappa_{0} \eta_{0}}+\frac{1}{\kappa_{1} \eta_{1}}\right) \int_{0}^{\infty} r^{\prime} q_{10}\left(r^{\prime}, f\right) J_{0}\left(\beta r^{\prime}\right) d r^{\prime} \\
& \quad=B_{1}(\beta, z=0, f) .
\end{aligned}
$$

The integral in Eq. (19) is simply the Hankel transformation of $q_{10}\left(r^{\prime}, f\right)$ to $q_{10}(\beta, f)$. Then,

$$
q_{10}(\beta, f)=\frac{\kappa_{1} \eta_{1} \kappa_{0} \eta_{0}}{\kappa_{1} \eta_{1}+\kappa_{0} \eta_{0}} B_{1}(\beta, f) .
$$

We could then transform $q_{10}(\beta, f)$ back to $q_{10}\left(r^{\prime}, f\right)$ via the inverse Hankel transformation but, as we shall see later, it is more convenient to retain the above form. Note that Eq. (20) constitutes a complete solution for the temperature anywhere in Hankel space, as the temperature at any point in both the air and the sample can be readily evaluated from Eq. (17) once the heat fluxes $q(\beta, f)$ are known. One numerical integration is required to express this result in real space; however, the integration is not difficult as $q(\beta)$ falls to zero as $\exp \left(-\beta^{2}\right)$ such that the integrand is effectively nonzero only over a relatively small range of $\beta$ values.

\section{EXTENSION TO MULTILAYER SOLUTIONS}

For the case of a sample consisting of a semi-infinite substrate and $N$ layers, we may write the temperature (in Hankel space) in each region as follows: For the ambient,

$$
\begin{aligned}
T_{0}(\beta, z, f)= & \frac{\alpha_{0}}{\kappa_{0}} \int_{0}^{\infty} q_{10}\left(r^{\prime}, f\right) G_{0}\left(\beta, z, f \mid r^{\prime}, z^{\prime}=0\right) \\
& \times 2 \pi r^{\prime} d r^{\prime}
\end{aligned}
$$

for layer $j$,

$$
\begin{aligned}
T_{j}(\beta, z, f)= & \frac{\alpha_{j}}{\kappa_{j}} \int_{0}^{\infty} q_{j-1, j}\left(r^{\prime}, f\right) G_{j}\left(\beta, z, f \mid r^{\prime}, z^{\prime}=0\right) \\
& \times 2 \pi r^{\prime} d r^{\prime}+\frac{\alpha_{j}}{\kappa_{j}} \int_{0}^{\infty} q_{j+1, j}\left(r^{\prime}, f\right) G_{j} \\
& \times\left(\beta, z, f \mid r^{\prime}, z^{\prime}=d\right) 2 \pi r^{\prime} d r^{\prime}+B_{j}(\beta, z, f),
\end{aligned}
$$

and for the substrate,

$$
\begin{aligned}
T_{N+1}(\beta, z, f)- & \frac{\alpha_{N+1}}{\kappa_{N+1}} \int_{0}^{\infty} q_{N, N+1}\left(r^{\prime}, f\right) G_{N+1} \\
& \times\left(\beta, z, f \mid r^{\prime}, z^{\prime}=0\right) 2 \pi r^{\prime} d r^{\prime} \\
& +B_{N+1}(\beta, z, f) .
\end{aligned}
$$

Note the contributions in Eq. (21b) from heat fluxes through both the top and bottom of the layer. Now, the $r^{\prime}$ dependence of all Green's functions in Eq. (21) is of the form $J_{0}\left(\beta r^{\prime}\right)$, so all four of the above radial integrals have the form

$$
\int_{0}^{\infty} q\left(r^{\prime}, f\right) J_{0}\left(\beta r^{\prime}\right) r^{\prime} d r^{\prime}=q(\beta, f),
$$

which is the definition of the Hankel transformation. The temperature equations (21) may then be combined with Eqs. (7) and (22) to give

$$
\begin{aligned}
& T_{0}(\beta, z, f)=\frac{1}{\eta_{0} \kappa_{0}} \exp \left(-\eta_{0} z\right) q_{10}(\beta, f), \\
& T_{j}(\beta, z, f)=\frac{1}{\kappa_{j} d_{j}}\left(\frac{1}{\eta_{j}^{2}}+2 \sum_{m=1}^{\infty} \frac{\cos (m \pi z / d)}{\eta_{j}^{2}+m^{2} \pi^{2} / d^{2}}\right) q_{j-1, j} \\
& \times(\beta, f)+\frac{1}{\kappa_{j} d_{j}}\left(\frac{1}{\eta_{j}^{2}}+2\right. \\
& \left.\times \sum_{m=1}^{\infty} \begin{array}{c}
(-1)^{m} \cos (m \pi z / d) \\
\eta_{j}^{2}+m^{2} \pi^{2} / d^{2}
\end{array}\right) \\
& \times q_{j+1, j}(\beta, f)+B_{j}(\beta, z, f), \\
& \Gamma_{N+1}(\beta, z, f)=\frac{1}{\eta_{N+1} \kappa_{N+1}} \exp \left(-\eta_{N+1} z\right) \\
& \times q_{N, N+1}(\beta, f)+B_{N+1}(\beta, z, f) .
\end{aligned}
$$
tities $^{19}$

At this point, it is convenient to make use of the iden- 


$$
\begin{aligned}
& \frac{\pi \operatorname{coth}(\pi x)}{2 x}-\frac{1}{2 x^{2}}=\sum_{m=1}^{\infty} \frac{1}{x^{2}+m^{2}} \\
& \frac{\pi}{2 x \sinh (\pi x)}-\frac{1}{2 x^{2}}=\sum_{m=1}^{\infty} \frac{(-1)^{m}}{x^{2}+m^{2}}
\end{aligned}
$$

to reduce the summations in Eq. (23). The temperature of a layer evaluated at $z=0, d$ becomes

$$
\begin{aligned}
T_{j}(\beta, z=0, f)= & \frac{\operatorname{coth}\left(\eta_{j} d\right)}{\kappa_{j} \eta_{j}} q_{j-1, j}(\beta, f) \\
& +\frac{1}{\kappa_{j} \eta_{j} \sinh \left(\eta_{j} d\right)} q_{j+1, j}(\beta, f) \\
& +B_{j}(\beta, f, z=0), \\
T_{j}(\beta, z=d, f)= & \frac{1}{\kappa_{j} \eta_{j} \sinh \left(\eta_{j} d\right)} q_{j-1, j}(\beta, f) \\
& +\frac{\operatorname{coth}\left(\eta_{j} d\right)}{\kappa_{j} \eta_{j}} q_{j+1, j}(\beta, f) \\
& +B_{j}(\beta, f, z=d) .
\end{aligned}
$$

We may then enforce the continuity of temperature and normal heat fiux at the interfaces to determine $q_{10}$, the heat flux into the ambient region, from which the beam deflections will be calculated. The heat fiux matching condition gives the general result $q_{i j}=-q_{j i}$. Continuity of the temperature at the first interface (ambient/top of film) yields

$$
\begin{aligned}
\frac{1}{\eta_{0} \kappa_{0}} q_{10}(\beta, f)= & -\frac{\operatorname{coth}\left(\vec{\eta}_{1} d\right)}{\kappa_{1} \eta_{1}} q_{10}(\beta, f) \\
& +\frac{1}{\kappa_{1} \eta_{1} \sinh \left(\eta_{1} d\right)} q_{21}(\beta, f) \\
& +B_{1}(\beta, f, z=0)
\end{aligned}
$$

for all values of $\beta$. At the last interface (bottom film/ substrate), we find

$$
\begin{aligned}
& \frac{-1}{\kappa_{1} \eta_{1} \sinh \left(\eta_{1} d\right)} q_{10}(\beta, f)+\frac{\operatorname{coth}\left(\eta_{1} d\right)}{\kappa_{1} \eta_{1}} q_{21}(\beta, f) \\
& \quad+B_{1}(\beta, f, z=d) \\
& =\frac{-1}{\eta_{2} \kappa_{2}} q_{21}(\beta, f)+B_{2}(\beta, f) .
\end{aligned}
$$

At an interface between two layers, denoted regions $j$ and $j+1$, where layer $j+1$ lies below (nearer the substrate) layer $j$, continuity of the temperature requires

$$
\begin{aligned}
& \frac{\operatorname{coth}\left(\eta_{j+1} d_{j+1}\right)}{\kappa_{j+1} \eta_{j+1}} q_{j, j+1}(\beta, f)+\frac{1}{\kappa_{j+1} \eta_{j+1} \sinh \left(\eta_{j+1} d_{j+1}\right)} \\
& \times q_{j+2, j+1}(\beta, f)+B_{j+1}(\beta, f, z=0) \\
& =\frac{1}{\kappa_{j} \eta_{j} \sinh \left(\eta_{j} d_{j}\right)} q_{j-1, j}(\beta, f)+\frac{\operatorname{coth}\left(\eta_{j} d_{j}\right)}{\kappa_{j} \eta_{j}} q_{j+1, j} \\
& \quad \times(\beta, f)+B_{j}\left(\beta, f, z=d_{j}\right) .
\end{aligned}
$$

Equations (26) and (27) constitute a set of $N+1$ linear equations for $N+1$ unknown normal heat fluxes at the interfaces, where $N$ equals the number of films in the system. This system of equations can be compactly written as the following tridiagonal matrix equation:

$$
\begin{aligned}
& {\left[\begin{array}{ccccc}
W_{0}+U_{1} & -V_{1} & 0 & \cdots & 0 \\
-V_{1} & U_{1}+U_{2} & -V_{2} & \cdots & 0 \\
0 & -V_{2} & U_{2}+U_{3} & \cdots & 0 \\
\cdots & \cdots & \cdots & \cdots & -V_{N} \\
0 & 0 & 0 & -V_{N} & U_{N}+W_{N+1}
\end{array}\right]} \\
& \times\left[\begin{array}{c}
q_{10} \\
q_{21} \\
q_{32} \\
\cdots \\
q_{N+1, N}
\end{array}\right]=\left[\begin{array}{c}
B_{1}(z=0) \\
B_{2}(z=0)-B_{1}\left(z=d_{1}\right) \\
B_{3}(z=0)-B_{2}\left(z=d_{2}\right) \\
\cdots \\
B_{N+1}(z=0)-B_{N}\left(z=d_{N}\right)
\end{array}\right],
\end{aligned}
$$

with the definitions

$$
W_{i} \equiv \frac{1}{\eta_{i} \kappa_{i}}, \quad U_{i} \equiv \frac{\operatorname{coth}\left(\eta_{i} d_{i}\right)}{\eta_{i} \kappa_{i}}, \quad V_{i} \equiv \frac{1}{\eta_{i} \kappa_{i} \sinh \left(\eta_{i} d_{i}\right)} .
$$

For the two-body case, $U_{1}$ should be replaced with $W_{1}$, the term appropriate to the substrate, in which case solution of the above equation yields the previously obtained expression (20).

For any multilayered system, we can easily calculate both the temperature due to heat generated within each region in the system (named $B$ above) and the normal components of the heat fluxes $q_{i j}$ through all interfaces in the system. These two quantities completely determine the tempcrature anywhere in the system via Eq. (23). The above exact result is simple compared to nonlocal Green'sfunction-based multilayer calculations, such as that of Madison and McDaniel. ${ }^{9}$ We now proceed to calculate the beam deflections in skimming transverse-mode photothermal deflection experiments from these temperature results.

\section{EVALUATION OF INFINITESIMAL PROBE BEAM DEFLECTIONS}

To calculate the photothermal beam deflections for multilayers, we begin with the solution for the temperature at any point in the ambient above the sample, given as

$$
\begin{aligned}
T(r, z, f) & =\int_{0}^{\infty} T(\beta, z, f) \beta J_{0}(\beta r) d \beta \\
& =\int_{0}^{\infty} \frac{\beta \exp \left(-\eta_{0} z\right)}{2 \kappa_{0} \eta_{0}} q_{10}(\beta, f) J_{0}(\beta r) d \beta,
\end{aligned}
$$

where we have demonstrated in the previous sections methods by which $q_{10}(\beta, f)$, the normal component of the heat flux through the top surface of the sample into the ambient, can be calculated for arbitrary layered systems. The angular defiection of an infinitesimal probe beam passing through the heated region above the sample is given by ${ }^{3}$ 


$$
\mathbf{M}=-\frac{1}{n} \frac{d n}{d T} \int \nabla T(\mathbf{r}) \times d \mathbf{r},
$$

where the integral is taken along the path of the probe beam. We will follow a procedure similar to that of Jackson et al. ${ }^{11}$ to evaluate Eq. (31). We will assume the deflection of the beam is small compared to the path length and that the probe beam propagates along $\hat{y}$. Then,

$$
\begin{aligned}
\mathbf{M} & =-\frac{1}{n} \frac{d n}{d T} \int_{-\infty}^{\infty} \nabla T(\mathbf{r}) \times \hat{y} d y \\
& =\frac{1}{n} \frac{d n}{d T} \int_{-\infty}^{\infty}\left(\frac{\partial T}{\partial z} \hat{x}-\frac{\partial T}{\partial x} \hat{z}\right) d y .
\end{aligned}
$$

Now, $x, y$, and $z$ are independent variables, hence we may swap the order of differentiation and integration in Eq. (32). We then have

$$
\begin{aligned}
\mathbf{M}= & \frac{1}{n} \frac{d n}{d T}\left[\frac{\partial}{\partial z}\left(\int_{-\infty}^{\infty} T(\mathbf{r}) d y\right) \hat{x}\right. \\
& \left.-\frac{\partial}{\partial x}\left(\int_{-\infty}^{\infty} T(\mathbf{r}) d y\right) \hat{z}\right]
\end{aligned}
$$

A single integration of the temperature is required,

$$
\begin{aligned}
\int_{-\infty}^{\infty} T d y= & \int_{\beta=0}^{\infty} \frac{\beta \exp \left(-\eta_{0} z\right)}{4 \pi \kappa_{0} \eta_{0}} q_{10}(\beta, f) \\
& \times \int_{-\infty}^{\infty} J_{0}\left(\beta \sqrt{x^{2}+y^{2}}\right) d y d \beta \\
= & \int_{\beta=0}^{\infty} \frac{\exp \left(-\eta_{0} z\right)}{4 \pi \kappa_{0} \eta_{0}} q_{10}(\beta, f) \cos (\beta x) d \beta
\end{aligned}
$$

Evaluation of the necessary derivatives of Eq. (34) and substitution of the result into Eq. (33) yields

$$
\begin{aligned}
M_{\tan }(x, h)= & -\frac{1}{n} \frac{d n}{d T} \int_{\beta=0}^{\infty} \frac{\beta \exp \left(-\eta_{0} h\right)}{4 \pi \kappa_{0} \eta_{0}} \\
& \times q_{10}(\beta, f) \sin (\beta x) d \beta, \\
M_{\text {norm }}(x, h)= & -\frac{1}{n} \frac{d n}{d T} \int_{\beta=0}^{\infty} \frac{\exp \left(-\eta_{0} h\right)}{4 \pi \kappa_{0}} \\
& \times q_{10}(\beta, f) \cos (\beta x) d \beta,
\end{aligned}
$$

where $h$ is the distance between the probe beam and the surface of the sample. The subscripts tan and norm in Eq. (35) denote the tangential and normal components of the beam deflection, respectively. Each is a complex number, as both have components both in and out of phase with the modulation of the heating beam. Note that the deflections depend on the location of the probe beam $(x, h)$ where $x$ is the distance from the probe beam to the center of the heating beam, and $h$ is the height of the probe beam above the sample surface. Note also that all of the information about the sample thermal and optical properties is contained in $q_{10}(\beta, f)$, so that Eq. (35) is valid for any multilayer sample structure.
Equations (35a) and (35b) are Fourier transform integrals, with $\beta$ interpreted as a spatial frequency, so we can perform a spatial Fourier transform on both sides of Eq. (35) to eliminate the numerical integrations over $\beta$. The deflections as a function of the spatial frequency $\beta$ are then

$$
\begin{aligned}
& M_{\mathrm{tan}}(\beta, h)=-\frac{1}{n} \frac{d n}{d T} \frac{\beta \exp \left(-\eta_{0} h\right)}{4 \pi \kappa_{0} \eta_{0}} q_{10}(\beta, f), \\
& M_{\text {norm }}(\beta, h)=-\frac{1}{n} \frac{d n}{d T} \frac{\exp \left(-\eta_{0} h\right)}{4 \pi \kappa_{0}} q_{10}(\beta, f) .
\end{aligned}
$$

For the two-body case, we find that

$$
\begin{aligned}
M_{\mathrm{tan}}(\beta, h)= & -\frac{1}{n} \frac{d n}{d T}\left(\frac{n k P_{0} C_{1}}{16 \pi^{2} \lambda}\right) \frac{\beta \exp \left(-\eta_{0} h\right)}{\kappa_{0} \eta_{0}+\kappa_{1} \eta_{1}} \\
& \times\left(\frac{1}{\eta_{1}+2 \gamma}\right) \exp \left(\frac{-\beta^{2} r_{h}^{2}}{4}\right), \\
M_{\text {norm }}(\beta, h)= & -\frac{1}{n} \frac{d n}{d T}\left(\frac{n k P_{0} C_{1}}{16 \pi^{2} \lambda}\right) \frac{\eta_{0} \exp \left(-\eta_{0} h\right)}{\kappa_{0} \eta_{0}+\kappa_{1} \eta_{1}} \\
& \times\left(\frac{1}{\eta_{1}+2 \gamma}\right) \exp \left(\frac{-\beta^{2} r_{h}^{2}}{4}\right) .
\end{aligned}
$$

Equations (37a) and (37b) are identical in form to the result obtained for $\delta$-function absorption at the surface ${ }^{2}$ with the addition of the correction

$$
C(k, \beta, \alpha)=\frac{2 \gamma}{\eta_{1}+2 \gamma} \text {. }
$$

This correction term approaches unity as the extinction coefficient becomes large, corresponding to the limit in which all energy is absorbed at the surface. Aamodt and Murphy have numerically investigated the effects of this correction on PTD measurements on semi-infinite samples in some detail. ${ }^{20}$

Note that the Fourier transform into the spatial frequency domain allows one to transform the experimental data once and use the simple analytical expressions (37a) and (37b) to fit the experimental data, rather than performing the integrations in Eq. (35) numerically at each iteration of the fitting procedure, thereby reducing the required computer time by orders of magnitude. In this case, no numerical integrations are required, regardless of the number of layers and absorbing regions in the sample, and regression fits to large bodies of experimental data can be performed easily on a personal computer.

The above theory applies to the case in which the probe beam is of infinitesimal width. In the following section, we generalize the deflection expressions to describe probe beams of finite extent, and investigate the effect of four-quadrant detection on the measurement of PTD signals.

\section{GENERALIZATION TO FINITE PROBE BEAMS}

The above theory is presented specifically for probe beams of infinitesimal extent, and should be valid for finite probe beams provided the width of the probe beam is much 
less than the thermal diffusion length in the ambient. ${ }^{12}$ If this is not the case, the above theoretical expressions should be corrected for the finite width of the probe beam, as the probe beam will be distorted upon passing the sample, and the deflection of the subsequent distorted beam is not well defined. Several authors have calculated corrections to beam deflection theory for Gaussian probe beams of nonzero extent. ${ }^{11,12}$ The general approach is to divide the probe beam into infinitesimal elements and average the deflections over these elements, weighting cach element according to the Gaussian intensity profile of the probe beam. The result of this calculation is the insertion of a multiplicative correction factor $w\left(R_{p}, h, \beta\right)$ into the integrands of Eq. (35),

$w\left(R_{p}, h, \beta\right)=0.5 \exp \left(\frac{i \omega R_{p}^{2}}{8 \alpha_{0}}\right)\left[1+\operatorname{erf}\left(\frac{\sqrt{2} h}{R_{p}}-\frac{\eta_{0} R_{p}}{2 \sqrt{2}}\right)\right]$,

where $h$ is the height of the center of the probe beam above the sample surface, $\omega$ is the chopping frequency, and $R_{p}$ is the probe beam radius.

This approach is not appropriate for the case of fourquadrant detection, though, as only the portions of the probe beam that are deflected across a detector quadrant boundary contribute to the measured signal, and a simple weighted average over the cntirc probc bcam cross section does not physically describe the actual experimental configuration. In the actual experiment, each component of the probe beam that is deffected across a detector quadrant boundary contributes a square-wave pulse to the total signal, and it is the Fourier component of the sum of all of these square pulses at the chopping frequency that is the measured signal. Jackson et al. ${ }^{11}$ calculated the detector response for this case assuming the probe beam was not distorted in passing the sample, such that the entire Gaussian profile of the probe beam was shifted in position on the detector but unchanged in shape. This is equivalent to assuming that the defiections are equal for all components of the probe beam. Using this method of calculation, we find the detector signal for normal or tangential deflections to be given by

$$
S(M)=\pi R_{p}^{2} \operatorname{erf}\left(\frac{\pi R_{p} M}{\lambda}\right),
$$

where $M$ represents either the normal or tangential deflection, $R_{p}$ is the probe beam Gaussian radius, $\lambda$ is the wavelength of the probe beam, and $\operatorname{erf}()$ represents the error function. For typical values of $R_{p}, M$, and $\lambda$, the argument of the error function is quite small, such that the signal is effectively linear in the deflections, and no correction to the infinitesimal probe beam deflection equations previously derived is required. If the probe beam is significantly larger than the thermal diffusion length in the ambient such that the deflection of the components of the probe beam is not uniform across the probe beam profile, the Gaussian shape of the probe beam will be distorted upon passing the sample, Eq. (40) will no longer be valid, and a more sophisticated approach to the calculation of the four-quadrant detector response is required.
We have attcmpted to solve this problem by calculating exactly the intensity signal from the four-quadrant detector as a function of the sample properties, probe and heating beam radii, and sample-detector distance. Since it is the Fourier component of this signal at the frequency of heating beam modulation that we measure (not the angular deflection of the beam), this calculation should provide the most accurate means for the interpretation of experimental PTD data.

We first calculate the normal and tangential signals due to a probe beam of infinitesimal extent. We then convolve this result with the Gaussian intensity profile of the probe beam to obtain results for probe beams of finite extent. We assume the center of the heating beam strikes the sample at $(x, z)=(0,0)$, and the center of the probe beam passes the sample at $\left(x_{0}, h\right)$. We will use a second primed set of coordinates centered on the detector such that $x^{\prime} \equiv x+x_{0}, z^{\prime} \equiv z+h$. Consider, then, a component of the probe beam that initially strikes the detector at the point $\left(x^{\prime}, z^{\prime}\right)$ when there is no thermal deflection of the beam. When the heating laser is switched on, the beam will undergo angular deflections both parallel and normal to the surface of the sample, denoted $\widetilde{M}_{t}(x, z)$ and $\widetilde{M}_{n}(x, z)$, respectively. If the distance from the sample to detector is large compared to the maximum distance the beam is defiected at the detector (a very good approximation), the distance that the beam is deflected at the detector is given simply by the product of the sample-detector distance $L$ and the angular deflections $\widetilde{M}$.

We then define the normal deflection signal as the difference in signal between the top and bottom halves of the detector, and the tangential signal as the difference in signal between the right- and left-hand-side halves of the detector. Only components of the probe beam that are deflected across the boundary between halves of the detector will contribute to the deflection signals. Mathematically, this condition is written

$$
L\left|\widetilde{M}_{t}\left(x^{\prime}, z^{\prime}\right)\right| \geqslant\left|x^{\prime}\right|
$$

for nonzero tangential signal, and

$$
L\left|\widetilde{M}_{n}\left(x^{\prime}, z^{\prime}\right)\right| \geqslant\left|z^{\prime}\right|,
$$

for nonzero normal signal. Components of the probe beam that do not satisfy the above conditions cannot contribute to the deflection signals.

Next, for an infinitesimal component of the probe beam that satisfies one (or both) of the above conditions, the point at which this component strikes the detector will be described as a function of time by the following equations:

$$
\begin{aligned}
& x^{\prime}(t)=x^{\prime}(t=0)+\operatorname{Re}\left[L \widetilde{M}_{t}\left(x^{\prime}, z^{\prime}\right) \exp (-i \omega t)\right] \\
& z^{\prime}(t)=z^{\prime}(t=0)+\operatorname{Re}\left[L \widetilde{M}_{n}\left(x^{\prime}, z^{\prime}\right) \exp (-i \omega t)\right] .
\end{aligned}
$$

If the corresponding inequality (41) is satisfied for the coordinate $x^{\prime}$ or $z^{\prime}$, there will exist two times $t_{1}$ and $t_{2}$ during each period (defined by $\omega$ ) at which the probe 
beam component under consideration crosses the boundary between detector halves. As the boundaries between the detector halves are at $x^{\prime}=0$ and $z^{\prime}=0$, the times $t_{1}$ and $t_{2}$ are given as the solutions of

Tangential:

$$
x^{\prime}(t=0)+\operatorname{Re}\left[L \tilde{M}_{t}\left(x^{\prime}, z^{\prime}\right) \exp (-i \omega t)\right]=0,
$$

Normal:

$$
z^{\prime}(t=0)+\operatorname{Re}\left[L \tilde{M}_{n}\left(x^{\prime}, z^{\prime}\right) \exp (-i \omega t)\right]=0 .
$$

The solutions of the above equations (with $\widetilde{M}=M^{\prime}+i M^{\prime \prime}$ ) are

$$
t_{1,2}=\frac{2}{\omega} \tan ^{-1}\left(\frac{M_{t}^{\prime} \pm \sqrt{\left|\widetilde{M}_{t}\right|^{2}-\left(x^{\prime 2} / L^{2}\right)}}{\left(x^{\prime} / L\right)-M_{t}^{\prime}}\right),
$$

for the tangential case, and

$$
t_{1,2}=\frac{2}{\omega} \tan ^{-1}\left(\frac{M_{n}^{\prime} \pm \sqrt{\left|\widetilde{M}_{n}\right|^{2}-\left(z^{\prime 2} / L^{2}\right)}}{\left(z^{\prime} / L\right)-M_{n}^{\prime}}\right),
$$

for the normal case, where we may now write $x^{\prime}(t=0)$ as $x^{\prime}$ and $z^{\prime}(t=0)$ as $z^{\prime}$. Now, as synchronous (lock-in) detection is used, the signal measured will be the component of the previously mentioned square pulse at the fundamental frequency of the heating beam modulation. Fourier transforming the pulse wave form, we find the in-phase (cosine transform) and out-of-phase (sine transform) components of the signal (assumed to be of unit amplitude) to be

$$
S_{\delta}\left(x^{\prime}, z^{\prime}\right)=(1 / \pi)\left[\sin \left(\omega t_{2}\right)-\sin \left(\omega t_{1}\right)\right],
$$

for in phase, normal, or tangential, and

$$
S_{\delta}\left(x^{\prime}, z^{\prime}\right)=(1 / \pi)\left[\cos \left(\omega t_{1}\right)-\cos \left(\omega t_{2}\right)\right],
$$

for out of phase, normal, or tangential. If we define the complex signal $\widetilde{S}_{\delta}\left(x^{\prime}, z^{\prime}\right) \equiv S_{\delta}$ (in phase) $+i S_{\delta}$ (out of phase) and insert the above expressions for $t_{1}$ and $t_{2}$ into the signal equations (45a) and (45b), we find (after some trigonometric manipulation),

tangential:

$\widetilde{S_{\delta}}\left(x^{\prime}, z^{\prime}\right)=\frac{1}{\pi \widetilde{M}_{t}\left(x^{\prime}, z^{\prime}\right)} \sqrt{\left|\widetilde{M}_{t}\left(x^{\prime}, z^{\prime}\right)\right|^{2}-\left(x^{\prime 2} / L^{2}\right)}$,

normal:

$\tilde{S_{\delta}}\left(x^{\prime}, z^{\prime}\right)=\frac{1}{\pi \widetilde{M}_{n}\left(x^{\prime}, z^{\prime}\right)} \sqrt{\left|\widetilde{M}_{n}\left(x^{\prime}, z^{\prime}\right)\right|^{2}-\left(z^{\prime 2} / L^{2}\right)}$.

The PTD signal measured as a function of $x_{0}$ and $h$ for probe beams of finite extent will then be given by

$$
\begin{aligned}
\tilde{S}_{t}\left(x_{0}, h\right)= & \int_{-\infty}^{\infty} \int_{x_{1}\left(z^{\prime}\right)}^{x_{2}\left(z^{\prime}\right)}\left[\frac{1}{\pi \widetilde{M}_{t}\left(x^{\prime}, z^{\prime}\right)}\right. \\
& \left.\times\left(\sqrt{\left|\widetilde{M}_{t}\left(x^{\prime}, z^{\prime}\right)\right|^{2}-\frac{x^{\prime 2}}{L^{2}}}\right)^{1 / 2}\right] \\
& \times \exp \left(\frac{-\left(x^{\prime 2}+z^{\prime 2}\right)}{R_{p}^{2}}\right) d x^{\prime} d z^{\prime},
\end{aligned}
$$

$$
\begin{aligned}
\widetilde{S}_{n}\left(x_{0}, h\right)= & \int_{-\infty}^{\infty} \int_{z_{1}\left(x^{\prime}\right)}^{z_{2}\left(x^{\prime}\right)}\left[\frac{1}{\pi \tilde{M}_{n}\left(x^{\prime}, z^{\prime}\right)}\right. \\
& \left.\times\left(\sqrt{\left|\widetilde{M}_{n}\left(x^{\prime}, z^{\prime}\right)\right|^{2}-\frac{z^{\prime 2}}{L^{2}}}\right)^{1 / 2}\right] \\
& \times \exp \left(\frac{-\left(x^{\prime 2}+z^{\prime 2}\right)}{R_{p}^{2}}\right) d z^{\prime} d x^{\prime}
\end{aligned}
$$

where $x_{1}\left(z^{\prime}\right)$ and $x_{2}\left(z^{\prime}\right)$ are the two roots of Eq. (42a) that represent the points furthest from the detector quadrant boundary that can still be deflected across that boundary, and $z_{1}\left(x^{\prime}\right)$ and $z_{2}\left(x^{\prime}\right)$ are the two roots of Eq. (42b) that represent the similar limits for the normal deflections. The dependence of $\widetilde{S}_{n}$ and $\widetilde{S}_{t}$ on $x_{0}$ and $h$ comes from the coordinate transformation between the primed coordinates (centered on the detector) and the unprimed coordinates, centered on the heating laser beam. The above integrals are intractable, and we have as yet to find useful approximations in which they can be evaluated. They are not expected to converge to the infinitesimal beam expressions $\widetilde{M}_{n}$ and $\widetilde{M}_{t}$ in the limit $R_{p} \rightarrow 0$, as an infinitesimal probe beam incident initially on the center of the four-quadrant detector will produce the same signal for any nonzero deflection of the beam. The above integrals do reduce to the expression derived above $\mathrm{Eq}$. (40) for the case of constant deflections over the entire probe beam profile. We are currently attempting numerical evaluations of the effects of the above corrections to the infinitesimal expressions, but the above integrals are quite difficult to evaluate numerically, as the limits on the inner integration must be calculated by solving Eq. (42) for each point of the outer integral.

\section{SAMPLE CALCULATIONS}

To demonstrate the usefulness of the above formalism we have performed several example calculations for PTD experiments. We perform these calculations for a two-layer system of aluminum nitride on aluminum on glass. The glass substrate is assumed to have the following properties: mass density $\rho=0.0025 \mathrm{~kg} / \mathrm{cm}^{3}$, specific heat capacity $C$ $=750 \mathrm{~J} /(\mathrm{kg} \mathrm{K})$, thermal conductivity $\kappa=0.0014 \mathrm{~W} /$ ( $\mathrm{cm} \mathrm{K}$ ), index of refraction $n=1.4$, extinction coefficient $k=0.0$. The aluminum film is fixed at $100 \AA$ thickness and is assumed to have the following properties: $\rho=0.0025$ $\mathrm{kg} / \mathrm{cm}^{3}, C=720 \mathrm{~J} /(\mathrm{kg} \mathrm{K}), \kappa=2.37 \mathrm{~W} /(\mathrm{cm} \mathrm{K}), n=2.74$, $k=8.32$. The aluminum nitride layer is of variable thickness and is assumed to have the following properties: $\rho$ $=0.00225 \mathrm{~kg} / \mathrm{cm}^{3}, \quad C=720 \mathrm{~J} /(\mathrm{kg} \mathrm{K}), \quad \kappa=0.16 \mathrm{~W} /$ ( $\mathrm{cm} \mathrm{K}$ ), $n=2.25, k=0.005$. We have chosen this sample structure as an example of an experimental attempt to measure the thermal properties of the aluminum nitride thin film. We assume the following experimental parameters: wavelength of heating laser $=6470 \AA$, Gaussian radius of heating laser beam $R_{h}=50 \mu \mathrm{m}$, probe beam radius $R_{p}=0 \mu \mathrm{m}$, height of probe beam above sample $h=100 \mu \mathrm{m}$, diffusivity of ambient medium (air) $=0.225 \mathrm{~cm}^{2} / \mathrm{s}$. Figures 3 and 4 show the normalized amplitude of the tangential 


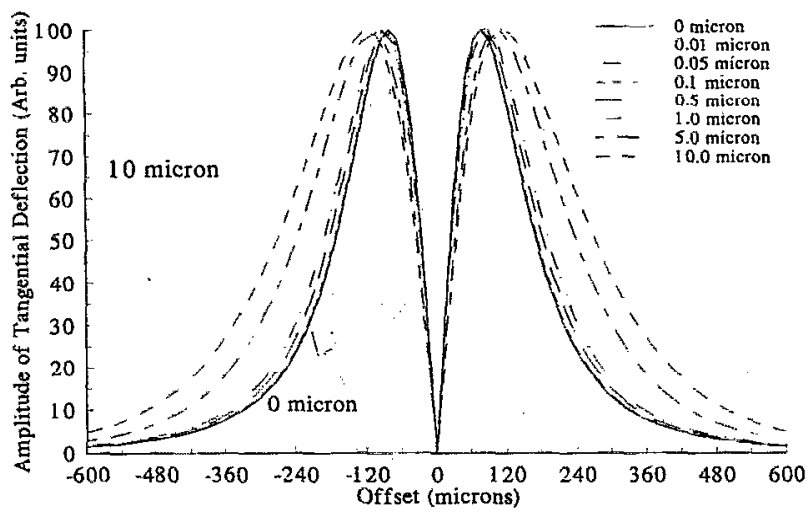

FIG. 3. Calculated amplitude of the tangential deflection for sample structure described in Sec. VIII. Data are calculated at a chopping frequency of $50 \mathrm{~Hz}$, and for aluminum nitride film thicknesses ranging from 0 to $10 \mu \mathrm{m}$.

deflection signal calculated for aluminum nitride film thicknesses ranging from 0 to $10 \mu \mathrm{m}$, for chopping frequencies of 50 and $1000 \mathrm{~Hz}$, respectively. Note the data is predicted to be insensitive to the existence of the aluminum nitride layer for thicknesses below about $0.1 \mu \mathrm{m}(1000 \AA)$. In Figs. 5 and 6 we show the sensitivity of the normalized amplitude of tangential deflection to a $10 \%$ change in the thermal conductivity of the aluminum nitride film, at chopping frequencies of 50 and $1000 \mathrm{~Hz}$, respectively. We see that reasonable sensitivity to the thermal conductivity of the aluminum nitride film can be expected for films 0.1 $\mu \mathrm{m}$ or more in thickness.

\section{SUMMARY}

In the present paper we report analytic expressions for temperature and probe beam deflection for multilayer systems based on Green's function techniques. Our results have several distinguishing unique features. First, the absorption of heat from the laser beam is treated analytically, and no approximations are required to obtain solutions for any number of layers. Any or all of the layers in the system, as well as the substrate, can have nonzero optical

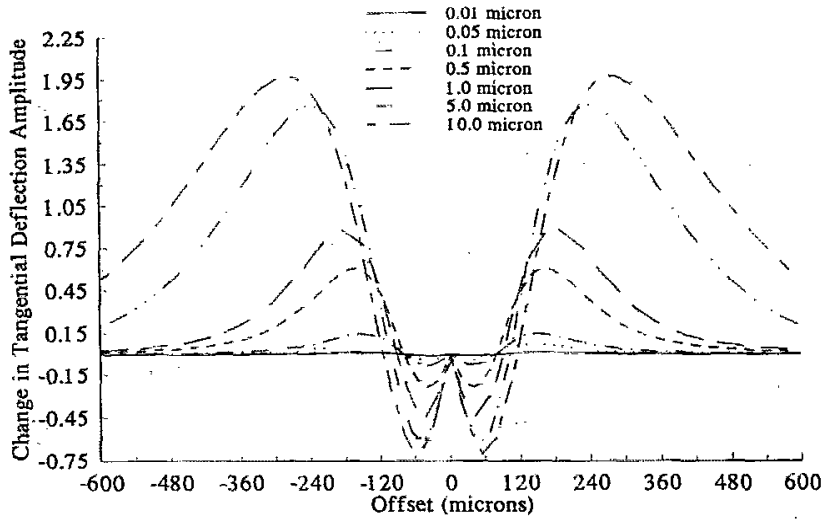

FIG. 5. Calculated change in normalized amplitude of the tangential deflection signal due to a $10 \%$ change in the thermal conductivity of the aluminum nitride layer in the sample described in Sec. VIII. Data are shown for aluminum nitride film thicknesses ranging from 0 to $10 \mu \mathrm{m}$, and are calculated for a chopping frequency of $50 \mathrm{~Hz}$.

absorption. Integrations over the heating distributions are performed analytically, such that numerical convolution as in the method of Grosse and Wynands ${ }^{12}$ is not required. Second, we use local Green's functions applied in Fourier transform space appropriate for steady periodic heating. By using local Green's functions we are able to treat the exact heating distribution in multilayers analytically, without numerical integrations or approximations to the heating profile. We use temperature and heat flux matching conditions between adjacent regions to link the local solutions. Such matching conditions were used by $\mathrm{Kant}^{8}$ and Iravani and Wickramasinghe, ${ }^{7}$ although they used only surface-source solutions for the temperature within each layer. Third, the central part of the calculation is a simple tridiagonal matrix equation for the boundary heat fluxes, such that the computing time required increases slowly with the number of layers in the system. This matrix equation is easily solved on a personal computer, even for many layers, and completely determines the temperature everywhere in the sample and the ambient, ${ }^{21}$ and completely determines the probe beam deflections. Also, if the photo-

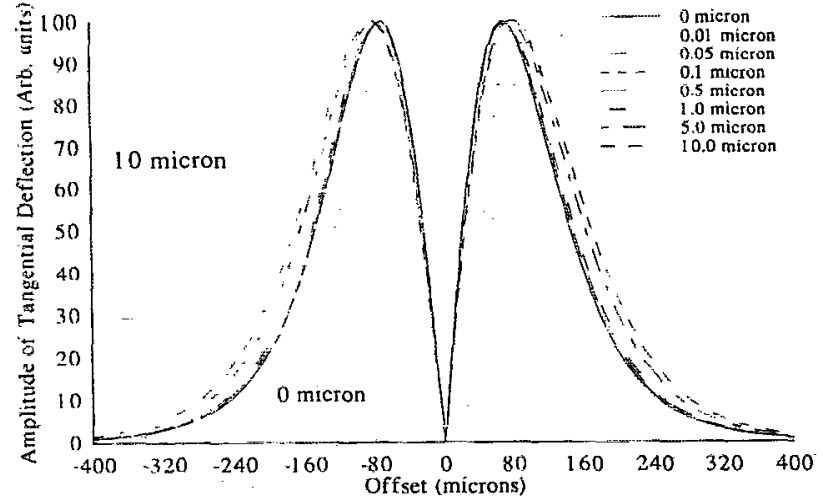

FIG. 4. Same as Fig. 3, but for a chopping frequency of $1000 \mathrm{~Hz}$.

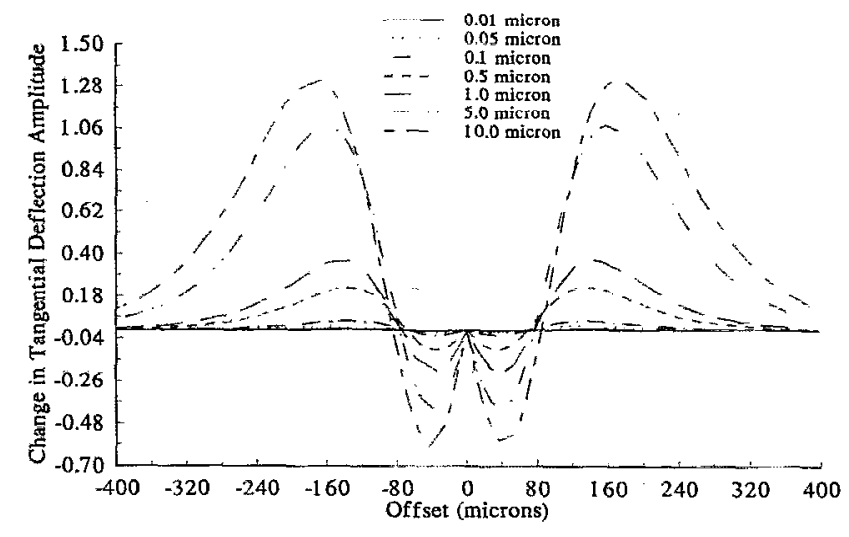

FIG. 6. Same as Fig. 5, but for a chopping frequency of $1000 \mathrm{~Hz}$. 
thermal deflection data is initially Fourier transformed, as is often done, ${ }^{22}$ the expressions for the deflections in Fourier space for any number of layers are free of numerical integrations, and our results are ideal for accurate analysis of experimental PTD data. Finally, we present new exact integral equations for the finite probe beam corrections for PTD experiments in which a four-quadrant detector is used. Analysis of experimental data will be reported in future papers.

\section{ACKNOWLEDGMENTS}

This research was supported by NASA Lewis Grants No. NAG-3-95 and No. NAG-3-154, and NSF Grant No. DMR8918889.

${ }^{1}$ P. K. Kuo, L. D. Favro, and R. L. Thomas, in Photothermal Investigations of Solids and Fluids, edited by J. A. Sell (Academic, Ncw York, 1988), Chap. 6, pp. 191-212.

${ }^{2}$ F. A. McDonald, Can. J. Phys, 64, 1023 (1986).

${ }^{3}$ L. D. Favro, P. K. Kuo, and R. L. Thomas, in Photoacoustic and Thermal Wave Phenomena in Semiconductors, edited by A. Mandelis (Wiley, New York, 1987), p. 70.

${ }^{4}$ M. Lax, J. Appl. Phys. 48, 3919 (1977).

${ }^{5}$ M. L. Burgener and R. E. Reedy, J. Appl. Phys. 53, 4357 (1982).

${ }^{6}$ R. J. Anderson, J. Appl. Phys. 64, 6639 (1988).
${ }^{7}$ M. Vaez Iravani and H. K. Wickramasinghe, J. Appl. Phys. 58, 122 (1985).

${ }^{8}$ R. Kant, J. Appl. Mech. 55, 93 (1988); R. Kant and K. L. Deckert, J. Heat Transfer 113, 13 (1991).

${ }^{9}$ M. R. Madison and T. W. McDaniel, J. Appl. Phys. 66, 5738 (1989).

${ }^{10}$ A. Mandelis and B. S. H. Royce, J. Appl. Phys. 50, 4330 (1979).

${ }^{11}$ W. B. Jackson, N. M. Amer, A. C. Boccara, and D. Fournier, Appl. Opt. 20, 1333 (1981).

${ }^{12}$ L. C. Aamodt and J. C. Murphy, J. Appl. Phys. 52, 4903 (1981).

${ }^{13}$ P. Grosse and R. Wynands, Appl. Phys. B 48, 59 (1989).

${ }^{14}$ M. Reichling, H. Grönbeck, and M. Schneider, in Proceedings of the 7th International Topical Meeting on Photoacoustic and Photothermal Phenomena, Doorwerth, The Netherlands, 1991.

${ }^{15}$ J. V. Beck, K. D. Cole, A. Haji-Sheikh, and B. Litkouhi, Heat Conduction Using Green's Functions (Hemisphere, New York, 1992).

${ }^{16}$ J. D. Jackson, Classical Electrodynamics (Wiley, New York, 1962).

${ }^{17}$ A. W. Crook, J. Opt. Soc. Am. 38, 954 (1948).

${ }^{18}$ K. E. Evans, A. N. Burgess, and R. A. McLean, Appl. Opt. 28, 328 (1989).

${ }^{19}$ I. S. Gradshteyn and I. M. Ryzhik, Table of Integrals, Series, and Products (Academic, New York, 1980).

${ }^{20}$ L. C. Aamodt and J. C. Murphy, J. Appl. Phys. 54, 581 (1983).

${ }^{21}$ K. D. Cole and W. A. McGahan, ASME 1992 Winter Annual Meeting, Anaheim, CA, November 1992 (to be published).

${ }^{22}$ T. R. Anthony, W. F. Banholzer, J. F. Fleischer, Lanhua Wei, P. K. Kuo, R. L. Thomas, and R. W. Pryor, Phys. Rev. B 42, 1104 (1990). 